\title{
Essais
}

\section{La Battaglia (1904-1913) : la revue des anarchistes italiens immigrés au Brésil}

La Battaglia (1904-1913): The review of Italian immigrant anarchists in Brazil

\section{Giovanni Stiffoni}

\section{(2) OpenEdition}

1 Journals

\section{Édition électronique}

URL : https://journals.openedition.org/essais/9055

DOI : 10.4000/essais. 9055

ISSN : 2276-0970

\section{Éditeur}

École doctorale Montaigne Humanités

\section{Édition imprimée}

Date de publication : 15 avril 2021

Pagination : 161-176

ISBN : 979-10-97024-09-3

ISSN : 2417-4211

\section{Référence électronique}

Giovanni Stiffoni, «La Battaglia (1904-1913) : la revue des anarchistes italiens immigrés au Brésil », Essais [En ligne], 17| 2021, mis en ligne le 27 avril 2021, consulté le 18 janvier 2023. URL : http:// journals.openedition.org/essais/9055; DOI : https://doi.org/10.4000/essais.9055 


\section{La Battaglia (1904-1913) : la revue des anarchistes italiens immigrés au Brésil}

\section{Giovanni Stiffoni}

Entre 1880 et 1920, plus d'un million d'immigrants italiens s'est installé au Brésil, grâce notamment aux favorables conditions offertes par les agences d'immigrations financées par le gouvernement brésilien ${ }^{1}$. C'est surtout à cette époque que les idéaux des mouvements sociaux ont commencé à se diffuser parmi les travailleurs brésiliens, par le biais de groupes de propagande et de périodiques. L'intérêt des historiens pour l'immigration des anarchistes italiens au Brésil s'explique par l'importance de l'activité politique réalisée par ce mouvement dans la nouvelle réalité sociale et politique. Citons par exemple l'expérience de la "Colonia Cecilia ", une communauté libertaire fondée en 1890 par le vétérinaire Giovanni Rossi. En outre, ce sont surtout les anarchistes qui se sont opposés au système politique autoritaire mis en place au Brésil, avant que Getulio Vargas essaie de "normaliser » la lutte sociale au Brésil. En dépit du rôle important qu’ont conservé les anarchistes italiens au Brésil, les secteurs politiques et économiques dominants dans le pays ont tenté de créer et de propager la thèse selon laquelle l'anarchisme serait une " plante exotique " qui n’aurait pas dû voir le jour dans un pays riche et prospère comme le Brésil ${ }^{2}$.

Les chercheurs qui ont souhaité mieux comprendre ces phénomènes sociaux ont aussi analysé l'instrument de communication le plus puissant utilisé à cette époque pour divulguer les idées anarchistes : le journal La Battaglia. En effet, ce périodique, pendant sa longue période de publication (1904-1913), a été distribué entre 3000 et 5000 exemplaires de chaque numéro, un volume qui s'avère considérable compte tenu de la répression policière constante à laquelle les militants anarchistes ont dû faire face sur ce territoire.

1 Angelo Trento, Do outro lado do Atlântico. Um século de imigração italiana ao Brasil. São Paulo: Nobel, 1989.

2 Carlo Romani, «Anarquismo italiano, transnacionalismo e emigração ao Brasil: Contribuiçóes ao debate teórico ", in Critica histórica, juillet 2020, Alagoas, p. 11. 
Les premières recherches significatives sur ce journal ont été menées par l'historien Luigi Biondi au début des années 1990 pour comprendre les effets de la grande immigration italienne dans l'État de São Paulo³ ${ }^{3}$ Ce chercheur italien a étudié avec soin les différentes tendances politiques qui se sont développées au sein du mouvement ouvrier naissant dans ce nouveau contexte, en accordant une attention particulière à la tendance anarchiste. Cette première étude restera également un point de référence pour les chercheurs qui étudieront ensuite $L a$ Battaglia. Isabelle Felici, spécialiste de civilisation italienne, poursuit sur cette voie en essayant de faire republier tous les périodiques de l'idéologie anarchiste en langue italienne $e^{4}$. Dans cette même étude elle a également tenté d'approfondir l'étude de l'organisation des ouvriers italiens au Brésil.

En fait, ces dernières années, la vision politique des ouvriers italiens émigrés au Brésil a fait l'objet de plusieurs débats, surtout après que l'historienne Edilene Toledo 5 a voulu minimiser l'importance du rôle des anarchistes dans le mouvement ouvrier au Brésil, en soulignant la différence entre ces derniers et les syndicalistes révolutionnaires. La contribution de Thiago Bernardo de Oliver à ce débat a aussi été importante, car il a souligné, indépendamment de cette différenciation proposée par Toledo, la participation des anarchistes dans l'histoire du mouvement ouvrier ${ }^{6}$.

L'étude biographique de la vie d'Oreste Ristori, réalisée par l'historien Carlo Romani, est elle aussi une œuvre très significative en cela qu'elle permet de démontrer l'évolution idéologique du directeur de La Battaglia pendant sa longue expérience au Brésil ${ }^{7}$.

C'est pourquoi nous souhaitons, dans cet article, examiner l'évolution vécue par ces militants dans un nouveau contexte sociopolitique, en cessant de les considérer comme des militants dotés d'une vision idéologique complètement définie. Par conséquent, il est nécessaire de comprendre de quelle façon évolue la relation des immigrés italiens avec leur pays d'accueil et comment change leur vision politique dans ce nouveau contexte. Jusqu'à présent, les chercheurs qui se sont occupés de ce sujet les ont considérés presque exclusivement comme les représentants d'une idéologie politique européenne définie, sans tenir suffisamment compte de leur évolution.

3 Luigi Biondi, «La stampa anarchica in Brasile: 1904-1915 ", Mémoire de Maîtrise (Histoire), Université de Roma La Sapienza, Italie, Rome, 1994.

4 Isabelle Felici, «Les italiens dans le mouvement anarchiste au Brésil, 1890-1920 ", thèse de doctorat (italien), 1994.

5 Edilene Toledo, Travessias Revolucionarias, Campinas: Editora Unicamp, 2004.

6 Tiago Bernardon Oliveira, "Anarquismo, sindicatos e revolução no Brasil " (1906-1937). Niterói, thèse de doctorat (Histoire), Universidade Federal Fluminense, 2009.

7 Carlo Romani, «Oreste Ristori. Uma aventura anarquista ", São Paulo, Annablume, 2002. 
Il sera tout d'abord nécessaire d'examiner les problèmes analysés par les rédacteurs de La Battaglia puisqu'ils sont la conséquence directe du nouveau contexte dans lequel ils exercent leur activité politique. Nous allons ensuite essayer de comprendre les nouvelles caractéristiques définissant le militantisme anarchiste qui se développe au Brésil. Cette recherche se situe dans le droit fil d'une thèse consacrée à Camillo Berneri ${ }^{8}$.

\section{L'anarchisme italien dans un nouveau contexte}

Aux yeux des anarchistes italiens, le Brésil représente le territoire où leur projet politique pourrait se réaliser plus facilement. En théorie, l'État brésilien, en raison de son histoire, a comme caractéristique d'être moins structuré que l'État italien et laisserait pour cette raison plus de place aux anarchistes italiens pour développer leur utopie. À titre d'exemple, Oreste Ristori, directeur de La Battaglia, se présente, dans une lettre adressée au président de la République, comme un "citoyen du monde qui ne reconnaît pas les frontières » et qui espère pouvoir profiter de sa nouvelle réalité sociale9.

L'analyse des commentaires parus dans La Battaglia montre que ces militants ont exprimé une réaction plutôt négative à propos de leur expérience dans ce nouveau contexte politique. A cet égard, il est significatif qu'ils fassent référence au Moyen-Âge pour représenter la situation au Brésil. Alessandro Cerchiai, l'un des écrivains les plus actifs du journal, a recours à cette comparaison dans l'article "Nel Secolo delle luci » publié dans le dernier numéro de janvier 1906. Après avoir raconté l'histoire d'un voleur d'animaux ayant été tué par quatre sbires envoyés par un fermier, il verse dans la satire :

Ici, le Moyen-Âge refait surface : les seigneurs locaux, les Bravi, les moines inquisiteurs, les condottieres, les amours des cloittres fleurissent avec exubérance sur le sol brésilien. Le temps d'Ezzelino da Romano renaît, ses horreurs, ses humiliations se répètent [...] Don Abbondio devient l'ami des sicaires et la Perpetua, sa servante, travaille comme maquereau. Fortebraccio gouverne et défait ${ }^{10}$.

Pour décrire le Brésil, Cerchiai fait référence à une série de personnages qui font partie de l'histoire et de la littérature italienne. Il cite d'abord le capitaine Ezzelino da Romano, originaire de la région de Trévise, connu pour sa cruauté et mentionné par Dante dans la Divine Comédie : il reste dans l'enfer, plongé dans un fleuve de sang, coupable d'avoir exercé une terrible violence contre son voisin. L'anarchiste toscan mentionne également Don Abbondio, sa servante et les Bravi, les hommes de main du seigneur local, tous protagonistes négatifs

8 En 2012 j'ai soutenu ma thèse intitulée Camillo Berneri (1897-1937). Mythes, racines et réalités d'un intellectuel anarchiste, Bordeaux, Université Bordeaux Montaigne.

9 Oreste Ristori, «Al presidente della repubblica », La Battaglia, 04/09/1904, p. 1.

10 Alessandro Cerchiai, "Nel Secolo delle luci », La Battaglia, 28/01/1906, p. 1. 
des Fiancés. Ce roman historique, œuvre de l'écrivain Alessandro Manzoni, offrait l'image négative typique de l'Italie du XVII siècle, arriérée et dominée par les étrangers.

Cerchiai cite également Fortebraccio, un homme politique connu et capitaine de l'Italie centrale au XVe siècle. Il le choisit pour souligner la corruption et le manque de moralité au Brésil, car ce personnage historique a été représenté par la propagande guelfe comme un homme cruel et ennemi de Dieu. En fait, Fortebraccio, proche de l'empereur, a presque réussi à établir un État en Italie centrale, et est donc devenu un grand ennemi du pouvoir papal. Cerchiai a une image si négative de son nouveau pays qu'il craint qu'il soit injuste de le comparer à la pire période de l'histoire de l'Italie : « Nous citons le Moyen-Âge, mais peut-être l'avons-nous calomnié, parce que [le Moyen-Âge] était meilleur que cette horreur. "

Il est significatif que Cerchiai, un anarchiste italien du début du XXe siècle, utilise le Moyen-Âge pour critiquer le système social brésilien car c'est le stéréotype traditionnel utilisé par les Lumières italiennes pour critiquer le passé le plus ignorant et le plus inculte ${ }^{11}$. La difficile expérience de Cerchiai au Brésil semble même lui faire récupérer l'Italie comme point de référence pour sa vision politique.

Malgré tout, cet anarchiste toscan, comme d'autres immigrants italiens, conclut son discours en introduisant l'espoir d'un changement du contexte politique et social qui justifie son choix de rester au Brésil. Il affirme : «malgré notre scepticisme, nous espérons trouver une voie de salut : les crimes de cette bourgeoisie meurtrière, ses vices obscènes qui l'épuisent, finiront par la précipiter dans un chaos sans fond, le peuple se réveillera! $»^{12}$ À cet égard, Piero Cofani, un collaborateur de La Battaglia qui écrit ses articles depuis Piracicaba, est également intéressant :

C’est ici au Brésil que l'avidité débridée et l'oppression politique du capitalisme rendent la situation du prolétariat encore plus insupportable quailleurs. (...) Mais le Brésil est encore un environnement vierge pour nous. Il y a beaucoup à faire et beaucoup à défaire. ${ }^{13}$

Dans ce discours développé par Piero Cofani, il y a une critique sévère contre l'organisation capitaliste de la société brésilienne, qui condamne le prolétariat à une situation extrêmement difficile. Mais ce contexte permet aussi d'espérer un changement social radical : cette société n'est pas encore développée comme en Europe et laisse la place aux anarchistes pour réagir et pour organiser une autre réalité économique et sociale.

11 Salvatore Guglielmino, Il Sistema Letterario. Settecento, Verona: Mondadori, 1992.

12 Alessandro Cerchiai, "Nel Secolo delle luci », La Battaglia, 28/01/1906, p. 1.

13 Piero Cofani, "Al lavoro compagni ", La Battaglia, 25/09/1904, p. 3. 
Même dans les articles d'Oreste Ristori, généralement si critiques de la réalité politique brésilienne, apparait parfois la possibilité de changer la situation du pays et la réalité individuelle elle-même. Dans un article intitulé "Tutto è metafisica al mondo ", l'anarchiste toscan considère qu'il est nécessaire que lui et ses compagnons soient réalistes : " en fin de compte, nous sommes au Brésil et le Brésil est l'environnement le plus approprié pour effectuer une métamorphose $»{ }^{14}$ Selon Ristori, son pays d'accueil de l'époque, bien que gouverné par " la lie des prisons ", est aussi un environnement caractérisé par une extrême fluidité qui laisse la possibilité de réaliser une nouvelle société.

Dans certains articles de La Battaglia, la nature exceptionnelle du Brésil est bien soulignée : ses forêts vierges, sa végétation luxuriante et ses pierres précieuses. Dans ce contexte cependant, selon Alessadro Cerchiai, il n'est possible d'écrire que "l'hymne à la rébellion " : "Libres sont les oiseaux, libres sont les bêtes, mais ici l'homme exploite l'homme, l'homme tue l'homme, l'homme est l'esclave de l'autre homme. ${ }^{15}$

La position politique des immigrés italiens est un aspect difficile à comprendre pour les intellectuels vivant au Brésil. Sans doute le transfert de nombreux citoyens européens vers l'Amérique latine était-il un phénomène très important à l'époque, mais les journaux anarchistes n'ont pas encore analysé en profondeur les conséquences de cet événement. Dans le journal de Ristori, la signification de l'immigration entre l'Italie et le Brésil est devenue un sujet très important, même si l'existence de La Battaglia est une conséquence de cet événement. Il est normal d'accorder beaucoup d'importance à la brochure Contra a Imigração, publiée en 1906, mais avant cela, la même vision politique critique de ce phénomène était déjà répandue.

Dans un article publié en 1904, Ristori recommande à ses compatriotes de ne pas se laisser convaincre par la propagande qui tend à édulcorer l'image de l'Amérique :
Vous qui écoutez les proxénètes de l'immigration qui passent dans les villages pour parler d'un délicieux et charmant Eldorado, cherchant des victimes pour devenir les nouveaux ânes de bât, vous devez crier que leurs propriétaires ont déjà mangé l'Amérique, qu'ici en vérité on meurt de faim. ${ }^{16}$

Une des missions de La Battaglia semble avoir été de proposer une image plus réaliste du Brésil aux citoyens italiens qui, vivant dans une situation économique difficile dans leur pays, étaient prêts à accepter n'importe quelle proposition de travail. Souvent, l'arrivée de travailleurs italiens au Brésil était subventionnée

14 Oreste Ristori, "Tutto è metafisica al mondo », La Battaglia, 07/01/1906, p. 1.

15 Anna De Gigli, "Malinconie ", La Battaglia, 07/07/1907, p. 1.

16 Oreste Ristori, "L'inquisizione al Brasile », La Battaglia, 20/06/1904, p. 1. 
jusqu'à ce qu'elle soit momentanément interrompue par le décret Prinetti en 1902, lorsque les autorités italiennes interdirent cette pratique en raison de la situation pénible que les immigrants devaient endurer sur les lieux de travail $^{17}$.

Ristori, dans un article du 11 septembre 1904, nie le mythe du travail facile dans les terres brésiliennes avec des mots très clairs : "Il n'est pas vrai qu'ici j'ai du travail pour le monde entier. Il n'est pas vrai que le travailleur est bien payé. Il n'est pas vrai qu'ici il y a de bonnes garanties pour les étrangers ${ }^{18}$. Il accuse clairement les agences intermédiaires qui mentent et contactent les travailleurs italiens et il critique également les autres journaux qui ne nient pas ces légendes créées pour attirer la main-d'œuvre au Brésil. En fait, même ces premiers articles qui commentent les conditions difficiles provoquent déjà la réaction des journaux bourgeois. En particulier, le Diario Popular appelle à la répression de la diffusion de La Battaglia, qui nuit à l'image du Brésil et à son activité économique ${ }^{19}$.

Gigi Damiani, un des rédacteurs de La Battaglia, estime également nécessaire de développer une réflexion sur ce sujet, car la domination de la bureaucratie brésilienne dans la gestion de l'immigration favorise l'arrivée d'immigrants italiens dans le pays mais n'améliore pas sa situation économique. Contrairement à Ristori, l'anarchiste romain ne cherche pas tant à donner une mauvaise représentation de la situation politique et économique du Brésil, mais analyse plus en profondeur ce que devrait être le type de relation de l'immigré avec son pays d'accueil : " il faudrait qu'il s'attache à la terre, qu'il sache comment fertiliser notre terre, qu'il veuille vivre notre vie et devenir notre chair et non plus un élément d'expansion de tel ou tel gouvernement ou d'un peuple d'esclaves ou de parias. ${ }^{20}$

Cela serait nécessaire pour éviter que ne subsiste cette logique de division entre les races qui, selon le militant romain, facilite la diffusion de l'impérialisme : " l'impérialisme n'est pas latin, ni slave, ni germanique, c'est l'impérialisme. " Il est intéressant que dans ce discours, Damiani mette en avant les principes censés caractériser cette nouvelle humanité d'immigrants qui devrait cesser d'être " une sentinelle » d'une armée envahissante pour devenir une " individualité consciente » d'un citoyen qui vit dans un pays libre et civilisé.

La Battaglia souligne que non seulement les Italiens ont été victimes de la politique économique des fazendeiros brésiliens et de leur État, mais que de nombreux immigrés européens se trouvaient dans une situation dramatique. Par exemple, dans un article publié au début de 1906, Antonio Bossi rapporte qu’il a trouvé

17 Angelo Trento, Do outro lado do Atlântico. Um século de imigração italiana ao Brasil. São Paulo: Nobel, 1989.

18 Oreste Ristori, "Lavoratori d'Europa non venite al Brasile », La Battaglia, 11/09/1904, p. 1.

19 "Le nostre sferzate ", La Battaglia, 23/09/1904, p. 1.

20 Gigi Damiani, "Pro Brasil », La Battaglia, 18/07/1905, p. 2. 
des groupes d'Espagnols dans un état de pauvreté extrême ${ }^{21}$. Ils avaient également été attirés au Brésil par les promesses des agents d'immigration et devaient ensuite supporter une réalité économique bien différente de celle promise.

Ces nouvelles publiées dans La Battaglia faisaient partie d'un plan de sensibilisation de la classe ouvrière européenne à la réalité vécue par les immigrants au Brésil qui a conduit à la publication du livret Contra a Imigração. Dans la biographie consacrée à Oreste Ristori, écrite par Carlo Romani, la naissance de ce projet est reconstituée en analysant également le témoignage que l'anarchiste fut contraint de donner au délégué de la police João Baptista de Souza en décembre $1906^{22}$. Selon ce document, environ 300 personnes se sont réunies pour mener une initiative en faveur des colons étrangers : plus de 3000 exemplaires de ce document (qu'il avait lui-même rédigé et qui a commencé à être diffusé dans différentes villes) ont été imprimés.

La commission ayant animé ce projet était constituée d'anarchistes, de socialistes et de citoyens d'origine italienne en général qui voulaient réagir contre un nouveau plan politico-économique de l'élite brésilienne. En fait, en 1906, la proposition d'attirer des immigrants italiens au Brésil revint en force, après l'interdiction de l'immigration soutenue par le décret Prinetti.

La publication du Contra a Imigração en Italie en décembre 1906 provoqua la réaction et la répression des autorités italiennes qui tentèrent d'empêcher sa publication, mais durent également reconnaître qu'il n'y avait pas de conditions pour suspendre le décret Prinetti. Même l'État de São Paulo jugea nécessaire d'annoncer qu'une brochure d'Oreste Ristori intitulée "Contre l'immigration " avait été publiée en Italie. Cette publication, selon le journal de São Paulo, réfuterait les mythes répandus par les agences d'immigration pour convaincre les travailleurs italiens de se déplacer pour trouver un emploi au Brésil, et l'État italien penserait donc à améliorer ses contrôles de l'immigration dans le sud du Brésil ${ }^{23}$.

Il est important de souligner que cette brochure traite de nombreux sujets déjà présentés dans La Battaglia et d'autres journaux anarchistes publiés au Brésil. Selon l'historien Biondi, l'un des principaux mérites de l'activité développée par Ristori et ses camarades fut la tentative de freiner l'immigration constante de paysans italiens, portugais et espagnols afin d'empêcher le système productif brésilien de tirer profit de leur faiblesse économique. C'est seulement de cette façon que le directeur de La Battaglia espérait empêcher que la disponibilité constante de la main-d'œuvre ne maintienne le coût du travail dans les plantations de café à un niveau bas ${ }^{24}$.

21 Antonio Bossi, «Dalle Caienne brasiliane. Via Crucis interminabile ", La Battaglia, 21/01/1906, p. 4.

22 Carlo Romani, Oreste Ristori. Uma aventura anarquista. São Paulo: Annablume, 2002, p. 160.

23 Ibid., p. 163.

24 Luigi Biondi, "La stampa anarchica in Brasile: 1904-1915 ", Mémoire de Maîtrise, Rome, Université de La Sapienza, 1994. 
Bien que La Battaglia soit née principalement pour aborder des questions politiques dans le nouveau contexte dans lequel ils vivaient, ces militants anarchistes ont fini presque involontairement par aborder des questions d'un autre type et par représenter également un point de référence pour la communauté des immigrés italiens établie au Brésil. Cette fonction sociale de La Battaglia semble évidente dans la partie consacrée au courrier communautaire où sont souvent abordés des thèmes qui n'ont pas beaucoup de rapport avec des sujets politiques. Par exemple, un article dans lequel est débattue une polémique littéraire dans laquelle un professeur qui utilise un ton « comico-réaliste " pour provoquer un pharmacien originaire de la ville du poète D'Annunzio. ${ }^{25}$

Dans une autre lettre publiée dans l'espace consacré aux lecteurs, Ristori prend parti dans une affaire familiale : un résident italien de Santa Rita do Passo Quatro, une petite ville du Minas Gerais, avait pris la responsabilité de soutenir les neveux d'un parent et ce dernier l'a dénoncé pour agression. ${ }^{26}$

Ristori recommande que la générosité de ce lecteur de La Battaglia soit reconnue et, en général, il est soucieux de publier la note suivante pour intensifier les liens entre la communauté d'immigrés italiens présente dans l'État de São Paulo et son journal :

Les camarades et les amis de l'intérieur de l'État sont expressément invités à nous envoyer des lettres sur le mouvement ouvrier, sur les exploits des autorités, sur les infamies des employeurs et surtout sur les drames qui se déroulent dans les «fazendas ». Pour cela, il n’est pas nécessaire d'être alphabétisé. Il suffit d'envoyer quelques détails, quelques données générales, pour que la rédaction s'occupe du reste. ${ }^{27}$

Il est évident que Ristori veut donner plus d'importance aux sujets politiques et pour cela La Battaglia augmente l'espace offert aux thèmes sociaux de la vie de l'immigration italienne au Brésil. C'est ainsi que sont publiées plusieurs lettres d'Italiens vivant au Brésil qui veulent essayer de reprendre contact avec des amis ou des parents dont ils n'ont plus de nouvelles.

Il est très important d'analyser ces informations car elles nous permettent de comprendre certains aspects de la vie des Italiens immigrés au Brésil à cette époque. Ils se plaignent, par exemple, du comportement des cambistas qui étaient une figure très importante dans leur vie à São Paulo ${ }^{28}$. Ceux-ci profitaient de l'ignorance fréquente des immigrants récemment arrivés pour exiger des taux d'intérêt très élevés et c'est pourquoi ils furent définis comme des « usuriers » dans certaines lettres.

25 Pasquino, "Quisquiglie ", La Battaglia, 28/05/1909, p. 4.

26 Oreste Ristori, "Cose di questo mondo », La Battaglia, 28/05/1909, p. 4.

27 Oreste Ristori, «Importante ", La Battaglia, 25/09/1904, p. 1.

28 "Altro che strozzini », La Battaglia, 25/09/1904, p. 3. 
On critique également une institution traditionnelle de la société brésilienne et portugaise comme la Santa Casa da Misericórdia, dont la mission est de traiter et de soutenir les malades et les handicapés. L'auteur de "Carità borghese " avertit les femmes étrangères qu'elles ne pouvaient pas compter sur ce soutien car il n'y avait pas de personnel parlant la langue des immigrants. ${ }^{29}$ C'est pourquoi cette institution devint un véritable " abattoir de chair humaine ", où des médecins inexpérimentés prescrivaient des médicaments inappropriés en raison de leur incapacité à comprendre la langue des malades ou de leur manque d'expérience.

Un autre article dénonçait les mauvaises conditions des hôpitaux où étaient hébergées les personnes infectées. Comme souvent, l'analyse de la situation commence par un cas particulier : un médecin a causé la mort du fils d'un employé parce qu'il avait fait une série d'injections inappropriées, le garçon ne souffrant pas, comme le pensait le fonctionnaire, d'une maladie respiratoire ${ }^{30}$. Selon l'auteur anonyme de l'article, ces situations se sont souvent produites parce que des « espions » ont dénoncé leurs voisins comme étant infectés sans en être sûrs.

Il est significatif que certains articles aient également été consacrés à décrire l'attitude de certaines voyantes qui ont tenté de profiter de l'innocence des Brésiliens, mais aussi des immigrants. Un des auteurs de ces textes a même raconté un épisode vécu par un de ses amis qui croyait qu'il mourrait jeune après avoir rencontré une de ces dames. ${ }^{31}$ Enfin, l'auteur de l'article parvient à rassurer son camarade, en plaisantant sur la crédibilité de cette voyante, mais insiste sur le caractère dangereux de ces figures qui arrivent même à ressembler désormais à des prêtres.

Il semble évident que La Battaglia ne se limite pas à diffuser les positions politiques des rédacteurs, mais finit aussi par s'intéresser aussi à la vie quotidienne des immigrés italiens, en publiant de nombreuses lettres qui abordent des sujets qui sont également communs aux habitants de São Paulo. Comme nous l'avons vu, Ristori invite souvent des Italiens vivant au Brésil à apporter leur contribution afin de présenter leur point de vue sur leur travail et la situation sociale difficile dont ils souffrent.

Malgré les appels du directeur de La Battaglia, il est clair que le discours qui se déroule à travers les lettres des immigrés italiens dépasse les limites du débat politique et finit par maintenir vivantes les relations sociales entre les immigrés qui se retrouvent dans une réalité nouvelle, inconnue et souvent hostile. Ristori n'était probablement pas aussi conscient de l'importance de la

29 Bulgaro, "Carità borghese ", 07/01/1906, p. 2.

30 "Come si uccidono i bambini poveri ", La Battaglia, 28/01/1906, p. 2.

31 Lucifero, «Le streghe ", La Battaglia, 30/05/1909, p. 3. 
fonction sociale de son journal, car il y avait « de nombreux appels de la rédaction à ne pas se laisser entraîner par les cas individuels et à éviter de raconter des événements locaux qui n'ont rien à voir avec les problèmes du travail $»^{32}$.

Toutefois, il serait important de souligner que l'importance des journaux des immigrants ne dépend pas uniquement de leur valeur politique mais, comme l'affirme le sociologue de l'école de Chicago Robert Ezra Parker, ce type de lecture est " "like a general store in a rural Community" " ${ }^{33}$ pour les étrangers vivant dans un pays dont beaucoup ne connaissent pas la langue. C'est pourquoi, malgré les recommandations de Ristori, tous les collaborateurs de La Battaglia ont instinctivement abordé des questions qui les ont aidés à mieux comprendre le Brésil et la situation de la communauté italienne, inquiets de la possibilité que leurs compatriotes s'éloignent de la lutte politique.

En outre, Parker estime qu'un autre grand mérite de la presse des immigrés a été de faire connaître la culture de ces nombreuses communautés aux citoyens de ces grandes villes. Par conséquent, à l'avenir, il sera très important de comprendre dans quelle mesure ces journaux, bien que nés avec des objectifs politiques, ont pu remplir ces autres fonctions sociologiques.

\section{Redéfinir les valeurs des militants anarchistes au Brésil}

En dehors des sujets qui ont évidemment un rapport direct avec le nouvel environnement dans lequel le groupe des anarchistes italiens a développé sa vision politique (le nouveau pays, l'immigration entre le Brésil et l'Italie et le rôle différent du journal dans ce nouveau contexte), l'analyse de La Battaglia nous a permis de mieux comprendre certains aspects qui caractérisent leur personnalité au début du XXe siècle. Sans aucun doute, Ristori et ses compagnons manifestent constamment dans leurs articles un fort ressentiment contre la classe sociale qui domine la société grâce à son pouvoir politique et économique, empêchant les groupes sociaux les plus pauvres d'obtenir une plus grande justice sociale. À cet égard, on évoque le menu succulent avec lequel la modeste mairie d'Amparo a accueilli le président de l'État de São Paulo Albuquerque Lins. Le commentaire de l'auteur est très acide : «Demain, si ces malheureux, qui paient, au nom de leur patrie, pour cette fête, descendent dans la rue pour demander des améliorations, les messieurs qui ont profité de leurs économies les feront fusiller $»{ }^{34}$ Le contraste existant entre le luxe des puissants et la souffrance des pauvres qui se précipitent pour récupérer de la nourriture reste fort.

32 Luigi Biondi, "La stampa anarchica in Brasile: 1904-1915 ", Thèse de Láurea (História), Université de Roma La Sapienza, Italie, Rome, 1994, p. 89.

33 Robert Ezra Parker, The immigrant press and its control, p. 113.

34 "Come si trattano i nostri tutori. Cose di questo mondo ", La Battaglia, 02/05/1909, p. 4. 
Le même ton si critique se retrouve dans un bref article où l'auteur anonyme se demande "où finit l'argent que chaque municipalité collecte " à un moment où la population est affectée par des limitations économiques constantes. ${ }^{35}$ Dans La Battaglia, le cas de João Florindo, secrétaire d'État qui s'est enfui avec l'argent public, est également cité. Et ce ne serait pas un cas isolé : " N'êtes-vous pas tous d'accord pour dilapider le patrimoine public? Qu'importe si, dans les différentes institutions publiques, chacun vole d'une manière différente? " ${ }^{36}$ Non seulement les politiciens et les administrateurs de la République brésilienne sont accusés de malhonnêteté, mais parfois les articles de La Battaglia attaquent aussi les responsables dans leur pays d'origine.

Dans un article, La Battaglia souligne le scandale de la Banque de Rome à la fin du XIXe siècle, manifestant même une remarquable impatience envers les administrateurs actuels : "Il faut déplorer la domination des barbares : fraude, vol, extorsion, détournement de fonds, pillage constant des banques et du Trésor public. " ${ }^{37}$ La bourgeoisie de tout pays est considérée, dans le journal de Ristori, comme " des parasites bien vivants qui appartiennent à notre même espèce. ${ }^{38}$ La seule fonction de ces politiciens serait de profiter de sa condition de pouvoir sans vraiment participer au processus productif. Les tons et les langages utilisés par les auteurs de La Battaglia sont peut-être différents de ceux des médias contemporains avec une vision critique de notre société, mais le contenu et le sentiment de colère suscités par l'injustice sociale dominant la société semblent être très proches sur le plan idéologique.

Pour Oreste Ristori, "cette immoralité et ce pillage qui fonctionnent comme un système de gouvernement dans tous les organismes de la vie administrative ne peuvent être supprimés ou combattus par le simple remplacement d'un président de la république. " Le directeur de La Battaglia prend cette position lorsque Hermès de Fonseca se présente comme candidat à la présidence de la république, démontrant l'impuissance de la population dans ce système politique où la malhonnêteté des administrateurs prédomine.

En fait, l'hostilité avec laquelle ils manifestent contre le système parlementaire provoque également une controverse contre les socialistes réformateurs. Dans l'Ami du peuple, un périodique auquel ont également collaboré plusieurs écrivains de La Battaglia, le discours de l'homme politique socialiste Prampolini, prononcé au parlement et applaudi même par la droite, est ridiculisé. La nomination d'un ministre socialiste au titre de Cavaliere del lavoro a également suscité des critiques ironiques, puisque Prampolini serait obligé de respecter une série de normes jugées indignes d'un représentant du prolétariat.

\footnotetext{
35 "Come si sbafa ", La Battaglia, 23/05/1909, p. 3.

36 "Come si deruba il pubblico ", La Battaglia, 04/09/1904, p. 1.

37 "Un'immensa collezione di ladri ", La Battaglia, 04/09/1904, p. 3.

38 "Un esercito di Pidocchi", La Battaglia, 25/09/1904, p. 4.
} 
Dans La Battaglia, une attention particulière est accordée aussi au socialiste réformiste Antonio Piccarolo, qui, après avoir participé à la fondation de l'ISP en Italie et avoir occupé divers postes électifs au sein du parti, a émigré au Brésil en 1904 pour diriger l'Avanti ! ${ }^{39}$. En raison de son socialisme modéré et de son soutien au parlementarisme, ce qui l'a toujours caractérisé, il quitte le journal et crée Il Secolo en 1906 et le Centro Socialista Paulistano en 1908. Selon Ristori, quiconque voulait défendre et revendiquer les droits des travailleurs en participant au système parlementaire comme Piccarolo se comporterait de la même manière que " tous ces médecins, avocats... qui ne pouvaient pas trouver de place dans la bourgeoisie, mettaient la cravate rouge. $"^{40}$

Cerchiai est également convaincu que "le socialisme ne viendra jamais du parlement $»^{41}$. Ce qui est intéressant, c'est qu'il aborde les détails du fonctionnement démocratique dans ses articles, en citant des cas concrets de mauvais comportement des représentants du peuple. En ce sens, il fait référence à l'absentéisme des parlementaires qui se définissent comme "populaires" (socialistes, républicains, radicaux...) lors des votes nécessaires pour annuler le financement des congrégations religieuses. Ces représentants politiques, au contraire, participent aux votes des lois formulées pour réduire les conflits entre le capital et le travail. Ainsi, selon le militant toscan, ils occupent un espace politique qui pourrait être utilisé par des mouvements ayant de «nouveaux idéaux " $"$.

Cerchiai, dans La Battaglia, critique le socialisme qui fait confiance au système parlementaire présent dans plusieurs pays (Belgique, Italie, France et Autriche) pour ne pas soutenir les revendications des travailleurs. Ces derniers sont victimes des manipulations et des sophismes présents dans les discours des représentants réformistes, car en réalité " au milieu du peuple on ne trouverait pas le révisionnisme de Bernstein, si mal plagié par le socialiste Filippo Turati.»

Il est clair que la position critique des auteurs de La Battaglia à l'égard des socialistes réformistes dépend également du contexte politique italien. Comme Malatesta l'a déjà déclaré, lorsqu'il s'est élevé contre le congrès socialiste tenu en Suisse en 1897 :

Les socialistes démocratiques, animés par l'esprit de domination et de centralisation qui les anime, voudraient unir leur parti et le mouvement ouvrier dans une même institution, en subordonnant la résistance économique à la lutte électorale. ${ }^{43}$

39 Fabio Bertonha, Sob a sombra de Mussolini: os italianos de São Paulo e a luta contra o fascismo, 1919-1945, São Paulo: Anna Blume, 1999, p. 11.

40 Oreste Ristori, Tutto è metafisica al mondo, La Battaglia, 7 janvier 1906, p. 1.

41 Anna De Gigli «La mistificazione scientifica del socialismo », La Battaglia, 28 mai 1909, p. 1.

42 Mastr'Antonio (Cerchiai), "La democrazia all'opera », La Battaglia, 23/05/1909, p. 4.

43 Errico Malatesta, "Il congresso socialista Italiano in Svizzera », L'Agitazione, 18/06/1897, p. 132. 
En même temps, dans le contexte brésilien, loin des polémiques constantes qui caractérisent, selon le penseur Camillo Berneri, le débat libertaire en Italie, l'antiparlementarisme traditionnel des anarchistes italiens au Brésil pourrait facilement trouver des arguments pour renforcer leur propagande.

Comme le montre José Murilo de Carvalho dans sa vaste bibliographie, les mécanismes de la Première République étaient incapables de garantir les principes démocratiques nécessaires au respect des droits du peuple brésilien. Au contraire, dans ce système politique, le chef pouvait continuer à utiliser les mêmes astuces qui lui garantissaient le maintien au pouvoir à l'époque de l'empire : le leader se souciait de garder le plus grand nombre d'électeurs pour son chef sur la liste électorale, les sbires étaient chargés de menacer les électeurs des opposants politiques, et les honnêtes citoyens refusaient souvent de participer aux élections par peur d'être victimes de violences. En outre, selon Murilo de Carvalho, les Brésiliens n'ont pas suivi le processus de formation nécessaire pour devenir citoyens d'une république démocratique, car la société brésilienne est profondément marquée par la tradition esclavagiste qui a dominé le pays pendant plusieurs siècles ${ }^{44}$.

Dans ce contexte, les auteurs de La Battaglia donnent une image très critique du peuple brésilien. Dans certains articles, ils semblent attribuer les responsabilités de cette situation sociale dramatique également aux habitants du pays : "Chaque peuple a le gouvernement qu'il mérite et nous pensons que le Brésil ne peut être une exception à la règle générale ${ }^{45}{ }^{45}$ Le même Ristori affirme que le peuple brésilien pourrait trouver une issue à ces terribles conditions de vie, mais il semble "infecté par le bacille de l'imbécillité » ${ }^{46}$.

Dans certains articles, il semble que la situation sociale difficile dans laquelle vivent les travailleurs au Brésil dépende également de l'attitude que la population brésilienne adopte. Il ironise sur la joie des gens qui sont impressionnés par l'éclairage et les drapeaux qui caractérisent les fêtes religieuses et patriotiques alors que c'est la même population qui « met la main à la poche et paie ". Souvent, les auteurs de La Battaglia soulignent que les puissants profitent de la situation parce que «le peuple paie et se tait " ${ }^{47}$.

Parfois, ce sont les gens eux-mêmes qui protestent contre ceux qui tentent de dénoncer l'exploitation des travailleurs et leur ignorance : dans un article de La Battaglia, on raconte le cas d'un écrivain de gauche qui est agressé par les habitants de Curitiba pour avoir publié un article anticlérical. L'auteur justifie ce triste événement en rappelant « les nombreux siècles d'esclavage et

44 Jose Murilo Carvalho, Os Bestializados, Companhia das Letras, 1987.

45 Oreste Ristori, "La baraonda politica ", La Battaglia, 30/05/1909, p. 1.

46 Oreste Ristori, «La baraonda politica ", La Battaglia, 30/05/1909, p. 1.

47 "Come si sbafa ", La Battaglia, 30/05/1909, p. 3. 
d'ignorance dont [le peuple] a souffert $»^{48}$. La dure réalité dans laquelle vivent les immigrants italiens au Brésil dépend de la façon dont ils traitent les travailleurs brésiliens dans les entreprises. Luigi Malavasi, l'un des collaborateurs de La Battaglia, veut montrer que la responsabilité des tristes conditions de travail au Brésil « ne dépend pas des patrons mais du travailleur, que se passerait-il si tous les travailleurs refusaient de travailler gratuitement ? La réponse est très simple : payez le travailleur correctement ${ }^{49}$.

Cette lecture de la réalité brésilienne nous surprend beaucoup, en tenant compte de ce qui est la vision dominante du peuple parmi les penseurs libertaires : il suffit de se rappeler la position de Kropotkine, largement publiée par les immigrés italiens, qui exalte tant la fonction de la révolution populaire. Il est important de souligner que même les auteurs de La Battaglia ont besoin de prendre de la distance par rapport à leur propre position. Par exemple, dans un article, Ristori doit se justifier d'avoir adressé quelques critiques aux lecteurs brésiliens, en précisant que " nous n'avons rien contre la population brésilienne qui est complètement innocente et irréprochable $~^{50}$.

Les anarchistes italiens ont eu probablement cette attitude parce qu'il leur manquait une institution qui puisse défendre les droits des travailleurs dans un contexte aussi difficile. La position critique qu'ils adoptent à l'égard de la société brésilienne est la conséquence d'une distance culturelle entre ces immigrants, qui ont souvent fui leur pays pour des raisons politiques, et un pays encore profondément marqué par l'expérience dramatique de l'esclavage. Les anarchistes italiens ont fait preuve d'une plus grande compréhension à l'égard de leur peuple "victime des abus des puissants " car ils pouvaient facilement s'identifier à eux et avaient une meilleure connaissance des mécanismes de domination dont ils avaient également été victimes.

Il est significatif que cette même vision de la population brésilienne présentée dans La Battaglia semble également affecter les anarchistes portugais, puisque l'historien Claudio Batalha trouve une attitude similaire dans le journal anarchiste Voz do trabalhador. Dans un article du 15 juillet 1908, il est dit que la classe ouvrière au Brésil vit encore dans un " état d'indolence " et de "prostration horrifiante", comme si l'esclavage existait encore ${ }^{51}$. L'historien José Murilo de Carvalho fait également référence à cette même attitude,

48 "Bestie umane ", La Battaglia, 02/04/1905, p. 1.

49 Luigi Malavasi, «Ai graduati ", La Battaglia, 30/05/1909, p. 3.

50 Oreste Ristori, Le nostre sferzate, La Battaglia, 25/09/1904, p. 1.

51 Claudio Henrique de Moraes Batalha, «Formação da classe operária e projetos de identidade coletiva ", in Jorge Ferreira et Lucilia Delgado (éds), O Brasil Republicano: O tempo do liberalismo excludente - da Proclamação República à Revolução de 1930. Livro 1, Rio de Janeiro, Civilização Brasileira, 2008, p. 114. 
lorsqu'il cite un biologiste français qui a déclaré que "le Brésil n’a pas de peuple $»^{52}$. Il est évident que les immigrants européens de cette période ont du mal à analyser avec lucidité la complexité de la réalité sociale du Brésil.

Notre analyse thématique du journal La Battaglia nous a permis de comprendre des aspects du militantisme libertaire qui ne sont guère mis en évidence dans la presse italienne : la distance idéologique avec les socialistes déterminée par la stratégie parlementaire de ces derniers, le ressentiment contre la classe dirigeante des systèmes politiques et économiques, et la difficulté d'interagir avec une population qui n'est pas très intéressée par la vision sociale des anarchistes.

En outre, ce qui me semble très pertinent, c'est que si l'on compare le type de message présenté dans la propagande anarchiste en Italie avec les articles publiés dans les journaux italiens au Brésil, on constate que dans ce deuxième cas, le discours politique est beaucoup plus simple et plus direct. Les écrivains de La Battaglia semblent expliquer leur vision sans se soucier tant des conséquences et des polémiques qu'ils pourraient soulever dans leur propre champ idéologique. Cerchiai, par exemple, affirme, sans crainte d'être démenti par ses collègues écrivains, que les principes de son mouvement étaient :

1) transformation de la propriété privée en propriété collective ou commune ;

2) abolition de tous les privilèges de caste ou de classe ;

3) abolition de tout pouvoir : armée, magistrature, etc. ;

4) l'internationalisme de toutes les revendications, c'est-à-dire l'abolition politique et juridique des États. ${ }^{53}$

Dans ce discours clair et immédiat, toutes les nuances qui caractérisaient les discours des penseurs anarchistes italiens, tels que Malatesta, Fabbri ou Saverio Merlino, qui s'efforçaient de trouver des compromis possibles entre les différentes tendances de l'anarchisme, disparaissent.

Probablement les anarchistes italiens émigrés au Brésil se sont-ils sentis plus libres de s'exprimer dans un contexte différent, où le poids des autorités dans le camp libertaire était moins important. De plus, nous devons être conscients que la plupart de ces militants ont joué un rôle de second plan dans le mouvement anarchiste italien et ont eu une préparation intellectuelle plus modeste que les penseurs anarchistes que nous venons de mentionner. C'est pourquoi ces opinions nous semblent souvent particulièrement importantes, car elles sont plus représentatives de l'opinion qui existait chez la majorité des

52 Jose Murilo Carvalho, Os Bestializados, Companhia das Letras,1987, p. 66.

53 Anna De Gigli, «La mistificazione scientifica del socialismo », La Battaglia, 28/05/1909, p. 1. 
militants anarchistes lorsqu'ils ont adopté un tel point de vue politique. C'est pourquoi la pensée libertaire semble souvent prendre des traits plus concrets et plus réalistes dans ces journaux que dans les complexes discours de leurs collègues plus préparés.

\section{Giovanni Stiffoni}

Professeur remplaçant

Université Fédéral Fluminense (UFF)

\section{Résumé}

La Battaglia (1904-1913), revue des anarchistes italiens immigrés au Brésil au début du $\mathrm{XX}^{\mathrm{e}}$ siècle, a été dirigée par Oreste Ristori. Les rédacteurs de la revue critiquent le capitalisme brésilien, et dénoncent l'exploitation des immigrés italiens au Brésil pour décourager les futurs candidats à l'immigration. La revue critique aussi le système parlementaire, le socialisme réformateur, et la passivité de la population brésilienne.

\section{Mots-clés}

La Battaglia, anarchistes italiens, Brésil, immigration, exploitation capitaliste.

\section{Abstract}

La Battaglia (1904-1913), a journal of Italian anarchists who immigrated to Brazil at the beginning of the 20 $0^{\text {th }}$ century, was directed by Oreste Ristori. The journal's editors criticised Brazilian capitalism, and denounced the exploitation of Italian immigrants in Brazil to discourage future immigration. The magazine also criticises the parliamentary system, reformist socialism, and the passivity of the Brazilian population.

\section{Keywords}

La Battaglia, Italian anarchists, Brazil, immigration, capitalist exploitation. 